\title{
Ultrasound Based Intraoperative Brain Shift Correction
}

\author{
Javier González, \\ Darío Sosa-Cabrera \\ Center for Technology in Medicine \\ University of Las Palmas de \\ Gran Canaria, Spain \\ jgonzalez,dario@ctm.ulpgc.es
}

\author{
Mario Ortega, \\ Jose Antonio Gil \\ MedicLab \\ University Polytechnic \\ of Valencia, Spain \\ mario.ortega@uv.es,jgil@upv.es
}

\author{
Antonio Tristán, Emma Muñoz-Moreno, \\ Rodrigo de Luis-García, \\ Rubén Cárdenes \\ Image Processing Lab \\ University of Valladolid, Spain \\ emunmor,atriveg,rluigar,ruben@lpi.tel.uva.es
}

\begin{abstract}
Intraoperative brain deformation (brain shift) limits the accuracy of image-guided neurosurgery. Intraoperative Magnetic Resonance (MR) images can be used for its estimation and correction, but open magnet scanners are very expensive. We propose a methodology that uses intraoperative 3D ultrasound (US) images for the brain shift correction, and we propose to use efficient techniques in order to compensate the brain deformation in real time. In order to evaluate our method, a head phantom was designed and built that allows to simulate the brain deformation and to validate our methodology.
\end{abstract}

\section{INTRODUCTION}

Brain surgery operations, such as tumor resection, are a quite challenging procedures that requires a rigorous planning and image guided techniques during the operation in order to accurately find the position of structures inside the brain. In practice, during the operation, when the skull is opened (craniotomy), there exists a relative motion of the brain with respect to the skull (also called brain shift).

This deformation is a consequence of various combined factors: cerebrospinal fluid (CSF) leakage, gravity, edema, tumor mass effect, brain parenchyma resection or retraction, and administration of osmotic diuretics [1], [2]. Intraoperative measurements show that this deformation is an important source of error that needs to be considered [3]. The extent of brain shift depends primarily on the size of the craniotomy and the duration of the surgery. Brain shift can be significantly reduced by opening only a small drill-hole in the skull, as is typical of minimally invasive stereo-tactic procedures. If stereotaxis is to be used after a craniotomy, however, the brain shift should be measured and corrected to ensure sufficient positioning accuracy.

Over the last years, the development of real time 3D ultrasound (US) imaging has revealed a number of potential applications in image guided surgery as an alternative to intraoperative MR. The major advantages of 3D US over existing intraoperative imaging techniques are its low cost and simplicity of use. However, the automatic processing of US images has not gained the same degree of development as other medical imaging modalities, probably due to the low signal to noise ratio of US images.

The structure of this paper is as follows: in the next section we describe our proposed methodology for brain shift correction, then section III describes the registration algorithm applied, after that in section IV we validate our method using a real phantom and finally section $\mathrm{V}$ presents our conclusions.

\section{Brain Shift Correction Methodology}

The proposed brain shift correction system is as follows. We start from preoperative 3D US and MR images. Then, a set of landmarks are selected in both volumes. We will use two types of landmarks: moving landmarks (those that will be free to move and located in soft tissues) and fixed landmarks (not allowed to move and located in the skull). A common reference system for both modalities is obtained by rigid registration, let's call the transformation that maps preoperative MR to preoperative US: $T_{R, 1}$, and the transformation that maps intraoperative MR to intraoperative US: $T_{R, 2}$. Once the craniotomy is performed, the brain is deformed and intraoperative 3D US is acquired. These intraoperative US data are registered against the preoperative US data by means of a Thin Plate Splines (TPS) algorithm, that we will explain in the following section, using the aforesaid landmarks. Let's call this non rigid transformation $T_{n}$. With these transformations we can estimate the brain deformation, applying them to the preoperative MR data. The final estimated result is obtained using this transformation:

$$
M R_{\text {intra }}^{*}=T_{R, 2}^{-1}\left(T_{n}\left(T_{R, 1}\left(M R_{P r e}\right)\right)\right.
$$

as we illustrate in figure 1.

\section{NON-RIGID REGISTRATION INCORPORATING RIGID STRUCTURES}

In neurosurgery images, we find different anatomical structures, both rigid (skull) and soft (brain), and therefore we can not apply the same elastic transformation to all the image because the elasticity is not constant anywhere.

In order to deal with this problem we could construct a physical model for a specific patient that predicts the interaction between the different rigid and soft structures. But it is impossible to obtain an accurate solution and it will have a high computational cost and many parameters to determinate (mass, elasticity, and others mechanical properties) [4]. 


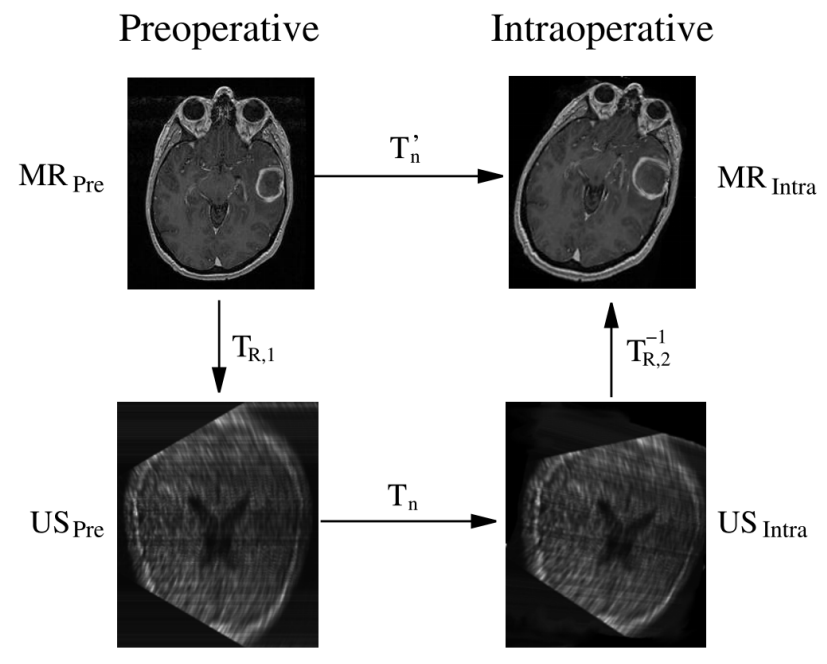

Fig. 1. Brain shift correction scheme of transformations

Several approaches have been proposed to deal with this problem. Rohr et al. [5] introduce an spline based elastic registration scheme. In this work, they consider the integration of anisotropic landmark errors as well as additional attributes at landmarks. As attributes they use orientations at landmarks using constraints through scalar products. They demonstrate that their scheme can preserve the shape of rigid structures embedded in elastic material.

Duay et al. [6] present an extension of the non-rigid registration Adaptive Basis Algorithm, that performs automatic segmentation of medical images even when structures have been displaced substantially. The algorithm automatically adjusts the stiffness of the transformation to permit large or small displacements depending on the stiffness defined over regions. The stiffness map can be defined once and for all in an atlas.

\section{A. Skull segmentation}

Our goal is to correct the deformation in real-time without deforming the rigid structure of the image (skull). For this task, it is necessary to identify the brain skull in the MR images, and then identify a set of landmarks on it that will be used as a constraint in the non-rigid transformation. For the segmentation of the bone's structure we use several methods:

- Isodata clustering for four regions [7].

- Mathematical morphology [8].

In Figure 2 we can see an example of the skull identification in a MR image.

It is important to reduce the identified landmarks at the skull in order to reduce the computational cost of the registration algorithm to keep our goal of real time computation. Notice that this cost depends on the number of landmarks because the computational complexity of the transformation function computation using thin plate splines is $O\left(N^{3}\right)$ with $N$ the number of landmarks. To reduce the number of points obtained at the segmentation phase we use:

- Homotopical Skull [8].

- Multi-resolution techniques.

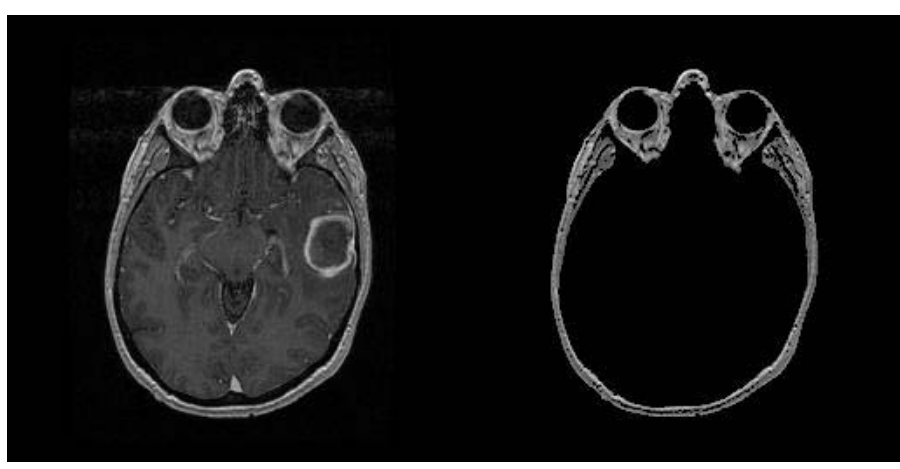

Fig. 2. Left: Original MRI, Right: Skull extraction of the MR image

- Add or delete landmarks at the skull depending of the distance at the deformation.

\section{B. Non rigid registration using Radial Base Functions}

One of the best known non-rigid registration methods is based on Radial Base Functions (RBF). The use of RBF in the medical image adjustment problem was originally introduced by Bookstein [9]. The RBF method is based on the use of control points or landmarks, so that if we identify a series of control points in both images (volumes), ensuring that these points are homologous, the problem is to determine the transformation function that allows the identification of any point between both images (volumes). In our case we try to obtain the transformation $T_{n}$ that maps the initial preoperative US data with the intraoperative US of the patient, using fixed landmarks obtained from the skull segmented in the preoperative MR volume ${ }^{1}$. This problem is equivalent to solve a problem of interpolation between the point sets: $X=\left\{\overrightarrow{x_{i}}=\left(x_{i}, y_{i}, z_{i}\right)\right\}_{i=1}^{N} \subseteq R^{3}$ (US preoperative moving landmarks + MRI skull fixed landmarks) and $Y=\left\{\overrightarrow{u_{i}}=\right.$ $\left.\left(u_{i}, v_{i}, w_{i}\right)\right\}_{i=1}^{N} \subseteq R^{3}$ (US intraoperative moving landmarks + MRI skull fixed landmarks). The transformation that maps one volume into the other: $T(x, y, z) \in C^{k \geq 0}$ fulfills:

$$
\overrightarrow{u_{i}}=T_{j}\left(\overrightarrow{x_{i}}\right), \quad i=1, \ldots, N
$$

The interpolation problem will be solved separately for each coordinate. In this case $T_{j}(x, y, z)$ indicates the displacement of variable $j=x, y, z$. If we use RBF we can be express this transformation as

$$
T_{j}(\vec{x})=\zeta_{j}(\vec{x})+\sum_{i=1}^{N} \alpha_{i, j} \psi\left(\left\|\vec{x}-\overrightarrow{x_{i}}\right\|\right), \quad j=x, y, z
$$

where $\psi(r)$ is the RBF function, $\alpha_{i, j}$ are coefficients to be found, and $\zeta_{j}(x, y, z)$ is an interpolation function with the following form:

$$
\zeta_{j}(\vec{x})=\beta_{1, j}+\beta_{2, j} x+\beta_{3, j} y+\beta_{4, j} z, \quad j=x, y, z
$$

${ }^{1}$ Notice that these fixed landmarks has to be transformed using $T_{R, 1}$ to have all the landmarks in the same reference system 


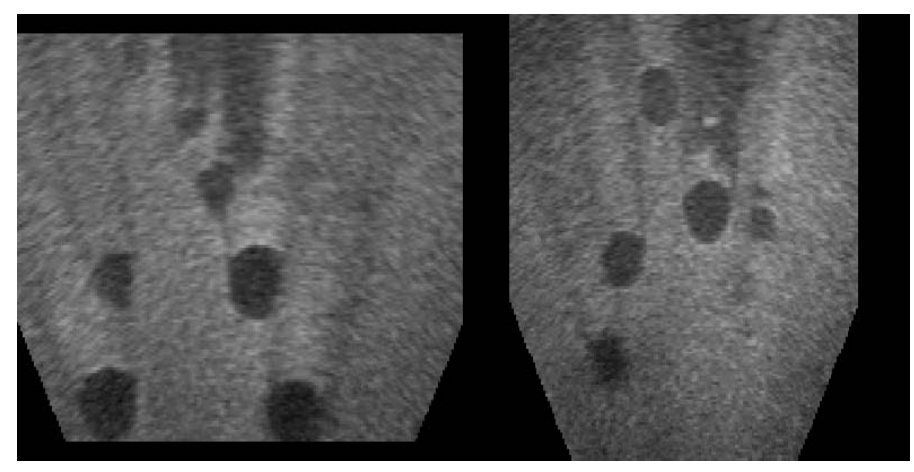

Fig. 3. Axial slice of the US volume of the phantom without pressure (left) and with pressure (right)

We need to impose the conditions of equation 2 , to finally obtain a linear equation system with $3(N+4)$ coefficients $(3 \mathrm{~N}$ for the radial part and 12 for the polynomial part) that can be solved very efficiently using LU decomposition.

In order to solve the interpolation problem the following RBF are usually used:

- Thin plate spline: $\psi(r)=r$.

- Multiquadratic: $\psi(r)=\sqrt{c^{2}+r^{2}}$, where $c \neq 0$ is a free selection parameter.

- $\psi(r)=e^{-c r^{2}}$ where $c>0$ is a parameter.

In the present research we have used the Thin Plate Spline approach, and we have guaranteed the solution of the equation system for the RBF previously described [10], [11].

\section{Validation Experiment using a Brain Phantom}

This method has been evaluated using a real phantom whose preparation is described below. The idea is to use this device to produce deformations similar to those produced in real cases when a craniotomy is done. A series of CT images of the phantom has been acquired when no pressure and when a given pressure is applied inside the phantom. We used CT for the validation study instead of MR, for technical reasons, that will not affect the final validation results. Acquisitions using 3D US in the same conditions have also been done, as shown in figure 3 where some of the balloons can be seen. Using these data sets we can compare the results obtained with our brain shift correction algorithm with the real CT images after deformation, in order to validate our method.

\section{A. Brain Phantom Container}

A glass-walled head phantom was devised, in which part of the surface was removed simulating a craniotomy, thus providing access to the brain for US imaging. An agar-gel solution, whose making is explained later, was introduced in the dummy in liquid state, and left in a refrigerator until solidification occurred. Ten fiducial balloons $5 \mathrm{~mm}$. in diameter were previously positioned inside the phantom using nylon threads. A balloon filled with $30 \mathrm{ml}$ of water was placed at the center and joined to a $50 \mathrm{ml}$ capacity syringe outside the dummy, through transparent flexible laboratory tube $4.8 \mathrm{~mm}$. in diameter. With this device, deformations to the gel phantom can be introduced, simulating intracranial pressure. After solidification, a suitable base was placed inside the neck of the dummy in order to limit the deformations of the simulated brain. The transparent dummy provides a convenient way for detecting fissures, heterogeneities and other abnormalities in the simulated tissue.

\section{B. Gel preparation}

Mean elastic modulus for the brain, as determined by [12], [13], was estimated to be approximately $8 \mathrm{KPa}$. In this manner, the amount of gel powder (Gelatin Gold DC, 200 Bloom) was calculated according to [14]:

$$
E_{\text {gel }}=0.003 \cdot C^{2.09}
$$

where $E_{g e l}$ is the desired Young's modulus of the gel, and $C$ is the gel concentration in grams per liter. Thus, a solution of de-ionized water $(2000 \mathrm{ml}$.) and $44 \mathrm{~g} / \mathrm{l}$. of pre-hydrated gelatin was heated and stirred until the temperature reached $60^{\circ} \mathrm{C}$. It was then placed into a water bath for cooling until the temperature decreased to $45^{\circ} \mathrm{C} .80 \mathrm{ml} / \mathrm{l}$. of n-propanol, $4 \mathrm{ml} / \mathrm{l}$. of $35-40 \%$ formaldehyde and $7 \mathrm{~g} / \mathrm{l}$. of agar powder were then added to the mixture. Formaldehyde was added to increase cross-linking among collagen fibers and raise the melting point of the gel [14], while n-propanol concentration increases sound speed [15]. At that time, glass microspheres were added in order to obtain appropriate scattering and absorption properties according to [14], [15]. The solution was then poured into the phantom container and let to rest in a refrigerator at $6{ }^{\circ} \mathrm{C}$ for approximately 12 hours. $150 \mathrm{ml}$. of the same solution were separated for subsequent mechanical elasticity testing, and poured into cylindrical moulds $5 \mathrm{~cm}$. in diameter. Young's modulus measurements were performed at room temperature using a computer-controlled servo testing machine integrating a $10 \mathrm{~N}$ load-cell, under similar testing conditions described in [14].

\section{Validation experiments}

We have used 6 moving landmarks representing the center of mass of 6 of the ten fiducial balloons placed inside the phantom, and 6 fixed landmarks, 4 of them near the craniotomy and 2 more in the opposite side of the skull near the nose. The fiducial balloons have been semi-automatically segmented in all the CT and US images to find the correct position of their center of masses. In our experiments we have applied the brain shift correction using the fixed landmarks and 5 moving landmarks between preoperative US and intraoperative US, and then we have checked the position of the one left out deforming the preoperative CT using the transformation obtained with the US. For that purpose, we used the transformation of equation 1. Numerical results are shown in table I, where it is clear that all the moving landmarks are better located after the correction, except landmark 6 . Notice that the correction is much better for landmarks 3, 4, and 5, but the errors do not decrease substantially for the others. The reason 


\begin{tabular}{|c|c|c|c|c|c|c|}
\hline Landmark number & 1 & 2 & 3 & 4 & 5 & 6 \\
\hline \hline Before correction & 4.301 & 2.685 & 2.981 & 2.937 & 2.350 & 2.283 \\
\hline After correction & 3.243 & 2.596 & 1.858 & 1.487 & 1.209 & 2.363 \\
\hline
\end{tabular}

TABLE I

ERRORS MEASURED IN MM FOR EVERY MOVING LANDMARK BEFORE AND AFTER THE BRAIN SHIFT CORRECTION

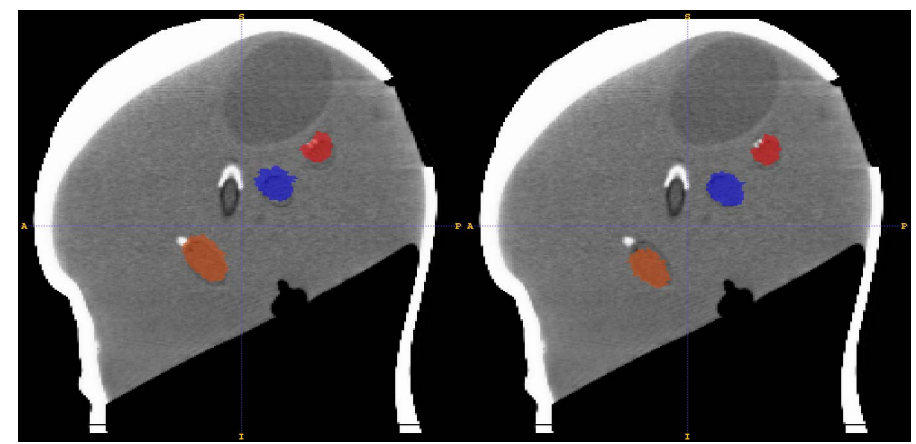

Fig. 4. Sagittal slice of the CT volume of the phantom, with overlapped balloon segmentations before (left) and after brain shift correction (right)

of this is because landmarks 3,4 , and 5 belong to interior balls, whose are surrounded by the other balls, and therefore their interpolation is better. The other landmarks are closer to the skull boundaries, and their correction is poorer because they are more affected by fixed landmarks in the skull. They will need more moving landmarks closer in order to achieve a better interpolation. Therefore the results will significantly improve with more landmarks, both fixed (on the skull) and moving ones (in the soft tissues).

We show in figure 4 the CT images of the deformed phantom overlapped with the segmented balloons before and after the brain correction. Let notice that two of the balloons shown in this figure are correctly aligned and the other one (the one at the left of the images) does not appear to improve its position too much, due to it is not a central balloon.

\section{CONCLUSIONS}

We have proposed here a method for brain shift correction using a landmarks based transformation algorithm that can be employed in real time. This method has been validated in a real phantom, that mimics the properties of real brain tissues, proving that our method can be successfully applied. It is important to highlight that perfect locations can not be obtained in our experiments using only 6 moving landmarks, but their locations can be substantially improved for interior landmarks. More accurate results could be obtained using more landmarks, and more work has to be done in order to show numerical results of that and the extra time required when the number of landmarks is increased.

One of the open questions in this methodology is the determination of the moving landmarks in the intraoperative US data and their correspondence with the landmarks selected in preoperative MR and US images. The landmarks in preoperative MR and US can be easily selected because there is no time restriction at that point, but once the craniotomy is done the intraoperative US data are acquired, and the corresponding landmarks have to be detected to perform the transformation. For that reason a fast automatic landmark detection method has to be developed to complete the whole methodology described here.

\section{ACKNOWLEDGMENT}

This work has been funded by the Comisión Interministerial de Ciencia y Tecnología, research grant TEC2004-06647-C0301 .

\section{REFERENCES}

[1] M. I. Miga, D. W. Roberts, F. E. Kennedy, L. A. Platenik, A. Hartov, K. E. Lunn, and K. D. Paulsen, "Modeling of retraction and resection for intraoperative updating of images." Neurosurgery, vol. 49, no. 1, pp. 75-84; discussion 84-5, Jul 2001.

[2] T. Hartkens, D. L. G. Hill, A. D. Castellano-Smith, D. J. Hawkes, C. R. Maurer, A. J. Martin, W. A. Hall, H. Liu, and C. L. Truwit, "Measurement and analysis of brain deformation during neurosurgery." IEEE Trans Med Imaging, vol. 22, no. 1, pp. 82-92, Jan 2003.

[3] J. Calvin R. Maurer, D. L. G. Hill, R. J. Maciunas, J. A. Barwise, J. M. Fitzpatrick, and M. Y. Wang, "Measurement of intraoperative brain surface deformation under a craniotomy," in MICCAI '98: Proceedings of the First International Conference on Medical Image Computing and Computer-Assisted Intervention. London, UK: Springer-Verlag, 1998, pp. 51-62.

[4] M. Ferrant, S. K. Warfield, A. Nabavi, F. A. Jolesz, and R. Kikinis, "Registration of 3D intraoperative MR images of the brain using a finite element biomechanical model," in MICCAI, 2000, pp. 19-28.

[5] K. Rohr, M. Fornefett, and H. S. Stiehl, "Spline-based elastic image registration: Integration of landmark errors and orientation attributes," Computer Vision and Image Understanding, vol. 90, no. 2, pp. 153-168, May 2003.

[6] V. Duay, P.-F. D’Haese, R. Li, and B. M. Dawant, "Non-rigid registration algorithm with spatially varying stiffness properties," in ISBI, 2004, pp. 408-411.

[7] F. R. D. Velasco, "Thresholding using the ISODATA clustering algorithm," IEEE Trans. Systems, Man and Cybernetics, vol. 10, no. 11, pp. 771-774, Nov. 1980.

[8] J. Serra and P. Soille, Eds., Mathematical Morphology and Its Application to Image Processing. Dordrecht: Kluwer, 1994.

[9] F. L. Bookstein, "Principal warps: Thin-plate splines and the decomposition of deformations," IEEE Transactions on Pattern Analysis and Machine Intelligence, June 1989, vol. PAMI-11, no. 6, pp. 567-585, 1989.

[10] B. S. Morse, T. S. Yoo, P. Rheingans, D. T. Chen, and K. R. Subramanian, "Interpolating implicit surfaces from scattered surface data using compactly supported radial basis functions," in Proceedings of the International Conference on Shape Modeling and Applications (SMI-01), B. Werner, Ed. Los Alamitos, CA: IEEE Computer Society, May 7-11 2001, pp. 89-98.

[11] M. Fornefett, K. Rohr, and H. S. Stiehl, "Radial basis functions with compact support for elastic registration of medical images," Image Vision Comput, vol. 19, no. 1-2, pp. 87-96, 2001. [Online]. Available: http://dx.doi.org/10.1016/S0262-8856(00)00057-3

[12] L. M. Bates and R. A. Robb, "Investigation of ultrasound image based correction of intraoperative brain shift," in 2nd IEEE international Symposium on Bioinformatics and Bioengineering BIBE, Washington, DC, 2001, pp. 254-261.

[13] Y. Fung, Biomechanics Mechanical Properties of Living Tissues. New York: Springer-Verlag, 1993.

[14] T. Hall, M. Bilgen, M. Insana, and T. Krouskop, "Phantom materials for elastography," IEEE Trans Ultrason Ferroelec Freq Contr, vol. 44, pp. 1355-1365, 1997.

[15] E. L. Madsen, J. Zagzebski, B. R.A., and R. Jutila, "Tissue mimicking materials for ultrasound phantoms," Med Phys, vol. 5, pp. 391-394, 1978. 